\title{
Isomeric Trimethylbenzyl Radicals Produced by Corona Discharge of 1,2,3,5-Tetramethylbenzene
}

\author{
Gi Woo Lee, ${ }^{\text {a }}$ Young Wook Yoon, and Sang Kuk Lee* \\ Department of Chemistry and The Chemistry Institute for Functional Materials, Pusan National University, \\ Pusan 609-735, Korea. *E-mail: sklee@pusan.ac.kr \\ Received June 11, 2011, Accepted June 30, 2011
}

\begin{abstract}
Isomeric trimethylbenzyl radicals were generated and vibronically excited from precursor 1,2,3,5-tetramethylbenzene, isodurene, with a large amount inert carrier gas helium in a corona excited supersonic expansion (CESE) using a pinhole-type glass nozzle. A long-path monochromator was used to record the visible vibronic emission spectra of the jet-cooled benzyl-type radicals in the $\mathrm{D}_{1} \rightarrow \mathrm{D}_{0}$ electronic transition. From the analysis of the spectra, we identified the evidence of the presence of three isomeric trimethylbenzyl radicals in the corona discharge, and obtained the electronic energy and a few vibrational mode frequencies in the ground electronic state for each isomer.
\end{abstract}

Key Words : Trimethylbenzyl radicals, Spectroscopy, Vibronic spectrum

\section{Introduction}

Whereas the benzyl radical, a prototypical aromatic radical, has been the subject of many spectroscopic studies, ${ }^{1}$ methylsubstituted benzyl radicals have received far less attention, especially for polymethylbenzyl radicals, due to the possible formation of several isomers from the precursor. $^{2}$

Spectroscopic works on benzyl-type radicals in the visible region have been performed by Schuler et $a l^{3}$ and Walker and Barrow. ${ }^{4}$ Bindley et al. ${ }^{5,6}$ have assigned several strong bands in the visible vibronic emission spectra of xylyl radicals produced from xylenes in a corona discharge. The technique of laser-induced fluorescence (LIF) was applied by Charlton and Thrush ${ }^{7}$ to obtain the vibronic assignments and lifetime measurement of xylyl radicals.

Hiratsuka et al. ${ }^{8}$ calculated the energies of close-lying doublet states, the $\mathrm{D}_{1}$ and $\mathrm{D}_{2}$ states in benzyl and xylyl radicals, and thereby described the vibronic coupling between two electronic states to explain the relaxation process. From the rotational contour analysis of the room temperature spectrum of the $o$-xylyl radicals, Cossart-Magos et al. ${ }^{9}$ determined the direction of the transition dipole moment change upon electronic excitation. High-resolution work has been also carried out by Fukushima and $\mathrm{Obi}^{10}$ who used the LIF technique to examine the vibronic coupling between the two lowest excited electronic states of the jet-cooled $p$-xylyl radical. Bandshape analysis of high-resolution emission spectra satisfactorily resolved controversial assignments of the $p$-xylyl radical. ${ }^{11}$ In addition, torsional analysis of the methyl rotor in xylyl radicals was conducted by Lin and Miller. ${ }^{12}$ Selco and Carrick assigned many bands in the vibronic emission spectra of benzyl and xylyl radicals. ${ }^{13,14}$

For polymethylbenzyl radicals, Lee group adapted an

${ }^{a}$ Present address: Department of Chemistry, Korea University, Seoul 136-701, Korea improved nozzle system coupled with corona discharge to observe the vibronic emission spectra of many species, ${ }^{15,16}$ from which each isomer generated from the precursor by removing a hydrogen atom from a different methyl group was clearly identified by analyzing the bandshape and the effect of methyl substitution on the electronic transition.

The effect of methyl substitution on the electronic properties has been well investigated for polymethylbenzyl radicals by analyzing fluorescence spectra. In particular, by using first- and second-order perturbation theory, Petruska ${ }^{17}$ derived general formulae showing that electronic transition of the parent hydrocarbon changes in intensity and shifts in frequency according to the nature, number, and position of the substituents on the ring. His results were generally consistent with the data obtained from the absorption spectra of these compounds.

The combination of corona discharge with supersonic expansion has been recognized as a powerful tool for observing the visible emission spectra of transient molecular species, especially for benzyl-type radicals. The spectral simplification and molecular stabilization associated with the expansion of a large amount of inert carrier gas cannot be achieved in any other way. Among the emission sources utilizing the combination, the pinhole-type glass nozzle provides sufficient continuous photon intensity for highresolution studies of weak transition in the gas phase and has been well adapted for observation of the vibronic emission spectra of benzyl-type radicals. ${ }^{18}$

In this study, we recorded the vibronic emission spectrum from the corona discharge of precursor 1,2,3,5-tetramethylbenzene, isodurene and identified the presence of three isomeric trimethylbenzyl radicals. In addition, we determined the spectroscopic data on the $\mathrm{D}_{1} \rightarrow \mathrm{D}_{0}$ electronic transition as well as the vibrational modes in the ground electronic state. 


\section{Experimental}

The experimental apparatus has been described in detail elsewhere. ${ }^{19}$ Briefly, the technique of corona excited supersonic expansion (CESE) was used to produce jet-cooled benzyl-type radicals in corona discharge by using a pinholetype glass nozzle. The vibronic emission spectrum was recorded with a long-path monochromator in the visible region.

Vibronically excited but jet-cooled benzyl-type radicals were produced from the corona discharge of precursor 1,2,3,5-tetramethylbenzene (Aldrich, reagent grade) seeded in a large amount of inert carrier gas helium. In order to improve the emission intensity, we increased the precursor concentration in the carrier gas by heating the sample temperature to $50{ }^{\circ} \mathrm{C}$ and adjusted the mixing ratio of helium to precursor by adjusting a bypass valve while monitoring the emission intensity from the strongest band, which was believed to be about $1 \%$ in the gas mixture. A pinhole-type glass nozzle with a $0.3 \mathrm{~mm}$ orifice diameter was adapted to eject the gas mixture in the corona discharge. A sharpened long stainless steel rod positioned inside the nozzle to act as the anode was connected to a high voltage dc power supply in a negative polarity, in which the axial discharging current was less than $5 \mathrm{~mA}$ at $1.5 \mathrm{kV}$ dc potential, and stabilized using a $150 \mathrm{k} \Omega$ current-limiting ballast resister.

A six-way cross-type Pyrex expansion chamber made of thick-walled $50 \mathrm{~mm}$ diameter glass tubes was evacuated by using a $800 \mathrm{l} / \mathrm{min}$ mechanical rotary vacuum pump to obtain a pressure of 3.0 mbar during continuous expansion, with 3.0 bar of backing pressure that was limited by the pressure tolerance of the glass materials. A long copper rod acting as a cathode was positioned parallel to the jet direction under the expansion chamber to prevent any arc noise from reaching the spectrometer.

A weak blue-green colored jet under the nozzle was the evidence for the presence of the benzyl-type radicals of the $\mathrm{D}_{1} \rightarrow \mathrm{D}_{0}$ transition in the expansion. The light emanating from the downstream jet area $5 \mathrm{~mm}$ below the nozzle opening was collimated by a collecting quartz lens $(f=5.0$ $\mathrm{cm}, \mathrm{D}=3.8 \mathrm{~cm}$ ) placed inside the chamber, focused onto the slit of the long-path monochromator (Jobin Yvon U1000) equipped with two 1800 lines/mm gratings, and detected with a cooled photomultiplier tube (Hamamatsu R649) and a photon counting system. During the scans, the slit was set to $100 \mu \mathrm{m}$, thereby providing an effective resolution of about 2 $\mathrm{cm}^{-1}$ in the visible region. The spectral region from 18000 to $22000 \mathrm{~cm}^{-1}$ was singly scanned at $2.0 \mathrm{~cm}^{-1}$ increment over 2 hrs to obtain the spectrum shown in Figure 2. The wavenumber of the spectrum was calibrated using the He atomic lines ${ }^{20}$ observed in the same spectral region as the benzyltype radicals, and was believed to be accurate within \pm 1.0 $\mathrm{cm}^{-1}$.

Since the vibrational modes of benzyl-type radicals have not been completely analyzed, $a b$ initio calculations were carried out on the ground $\left(\mathrm{D}_{0}\right)$ and lowest excited $\left(\mathrm{D}_{1}\right)$ electronic states of the isomeric trimethylbenzyl radicals to assist in the assignment of the electronic transition and the vibronic structure of each isomer produced. The calculations were executed with a personal computer equipped with an Intel Pentium IV CPU $2.80 \mathrm{GHz}$ processor with 2.0GB RAM, according to the standard method in the Gaussian program for Window package. The geometry optimization, vibrational frequency, electronic transition energy and oscillator strength calculations were performed at the time-dependent density functional theory (TD-DFT) level, and the $6-311 \mathrm{G}^{* *}$ basis set was employed in all of the calculations. The atomic motion of each mode was visualized using the HyperChem program with the output of the calculation.

\section{Results and Discussion}

It has been demonstrated ${ }^{21}$ that a well-controlled corona discharge of toluene seeded in a large amount of carrier gas produces jet-cooled benzyl radicals in the vibronically excited state. Alternatively, the use of benzyl fluoride as precursor produces much weaker emission intensity due to the large bonding energy of C-F. Although the mechanism for the generation and excitation of benzyl radical in corona excitation has not been exactly established, it has been suggested that the metastable He atom in the $1 \mathrm{~s} 2 \mathrm{~s}{ }^{3} \mathrm{~S}_{1}$ state, ${ }^{22}$ about $160,000 \mathrm{~cm}^{-1}$ above the ground state produced by corona discharge, transfers the excitation energy to the toluene through a collisional process, thereby producing a benzyl radical by removing a hydrogen atom from the methyl group rather than the benzene ring. The mechanism for the production of an $o$-xylyl radical from $o$-xylene has been well described in the liquid phase ${ }^{23}$ by laser experiment. The electronic excitation may loosen one of the $\mathrm{C}-\mathrm{H}$ bonds of the methyl group, eventually leading to the removal of a hydrogen atom from the methyl group.

Even though the benzyl-type diradical ${ }^{24}$ has been observed in the very cold solid matrix from 1,3,5-trimethylbenzene by eliminating a hydrogen atom from each of the two methyl groups, it has never been detected in the gas phase because of its extremely short lifetime and low concentration in the medium.

Since the molecule has a planar structure with 7 delocalized $\pi$ electrons to which the $\mathrm{CH}_{2}$ group contributes a $\pi$ electron, the electronic interaction between the methyl group and the benzene ring is undoubtedly much weaker than that between the methylene group and the benzene ring in the case of methyl-substituted benzyl radicals. Thus, the electronic structure of polymethylbenzyl radicals should exhibit a close relationship to that of the benzyl radical, and indeed one might be able to closely relate the two lowest excited electronic states of polymethylbenzyl radicals to the parental benzyl radical $2^{2} \mathrm{~B}_{2}\left(\mathrm{D}_{2}\right)$ and $1^{2} \mathrm{~A}_{2}\left(\mathrm{D}_{1}\right)$ states. Thus, the weak visible emission transition of polymethylbenzyl radicals is believed to arise from the close-lying $\mathrm{D}_{2}$ and $\mathrm{D}_{1}$ excited electronic states to the $1^{2} \mathrm{~B}_{2}\left(\mathrm{D}_{0}\right)$ ground state. Ring substitution is expected to affect the energies of the two excited electronic states, in which the electronic energy strongly depends on the nature, the position, and the number of the 
substituents on the benzene ring.

In benzyl-type radicals, two excited electronic states could be mixed through vibronic coupling which increases the relaxation rate of the population from $D_{2}$ to $D_{1}$ states. Therefore, the emission intensity of the $\mathrm{D}_{2} \rightarrow \mathrm{D}_{0}$ transition is inversely proportional to the density of the vibrational states in the $\mathrm{D}_{1}$ state. The separation of about $800 \mathrm{~cm}^{-1}$ between $D_{2}$ and $D_{1}$ states in the benzyl radical includes many vibrational levels, resulting in almost negligible emission intensity in the $\mathrm{D}_{2} \rightarrow \mathrm{D}_{0}$ transition while exhibiting strong intensity in the $\mathrm{D}_{1} \rightarrow \mathrm{D}_{0}$ transition. The only exception among benzyl-type radicals is the strong observation ${ }^{25}$ of the $p$-chlorobenzyl radical in the $\mathrm{D}_{2} \rightarrow \mathrm{D}_{0}$ transition, which could be attributed to the small difference of $94 \mathrm{~cm}^{-1}$ between the two excited electronic states.

Figure 1 shows the possible formation of benzyl-type radicals from the corona discharge of precursor 1,2,3,5tetramethylbenzene seeded in a large amount of inert carrier gas helium. Three isomeric trimethylbenzyl radicals could be generated by removing a hydrogen atom from the methyl group at different positions. Although the most trustworthy spectroscopic identification of large aromatic molecules of similar structure is obtained by analyzing a high resolution spectrum showing rotational structure, as in the routine spectroscopic analysis, the observation of the origin band of the electronic transition, as well as a few vibronic bands of well-known vibrational modes, may provide reliable evidence for the assignment of benzyl-type radicals formed in corona discharge. Thus, we attempted to obtain evidence of each isomer produced by comparing the vibronic bands measured from observation with the calculated bands for the electronic transition and vibrational modes of each isomer because the spectrum observed in this work showed a limited rotational temperature and resolution.

From the photolysis of 1,2,3,5-tetramethylbenzene in the solid state, Branciard-Larcher and Migirdicyan ${ }^{2}$ observed three maxima in fluorescence spectrum at 513.0, 489.0, and $477.0 \mathrm{~nm}$ and assigned them to the origin bands of 2,3,5-, 3,4,5-, and 2,4,6-trimethylbenzyl radicals, respectively, by comparing with the result of a simple linear combination of atomic orbital, self-consistent-field calculation. The calculation showed very good agreement with the observation for the electronic transition energy and oscillator strength of each isomer. The sequence order of origin bands in the $\mathrm{D}_{1} \rightarrow$ $\mathrm{D}_{0}$ transition among the three isomers was consistent with those predicted by substitution effect which states that the

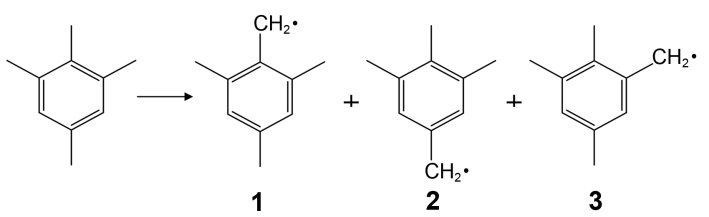

Figure 1. Possible formation of three isomeric trimethylbenzyl radicals by removal of a hydrogen atom from the methyl group at different positions in the corona discharge of the precursor 1,2,3,5tetramethylbenzene. The numbers $\mathbf{1}, \mathbf{2}$, and $\mathbf{3}$ in brackets represent 2,4,6-, 3,4,5-, and 2,3,5-trimethylbenzyl radicals, respectively.

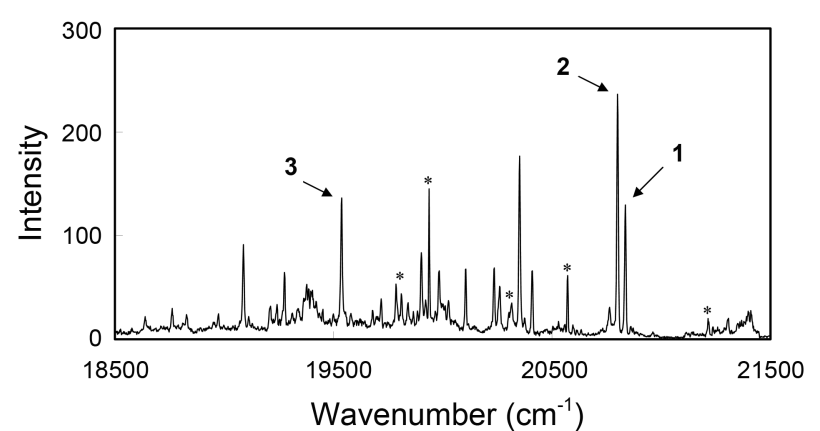

Figure 2. Visible vibronic emission spectrum observed from a corona discharge of precursor 1,2,3,5-tetramethylbenzene with a large amount of inert carrier gas helium in CESE. The origin bands of 2,4,6-, 3,4,5-, and 2,3,5-trimethylbenzyl radicals are indicated by the numbers $\mathbf{1}, \mathbf{2}$, and $\mathbf{3}$, respectively, in the spectrum. The atomic lines are indicated by an asterisk.

shift of the electronic transition depends on the nature, number, and position of the substituents on the ring. ${ }^{17}$ The effect was applied to the results of both difluorobenzyl and dimethylbenzyl radicals by counting the position of substitution.

Figure 2 shows a portion of the vibronic emission spectrum of the benzyl-type radicals formed by the corona discharge of precursor 1,2,3,5-tetramethylbenzene, in which many of the strong bands are observable with a very good $\mathrm{S} / \mathrm{N}$ in the region of $18500-21500 \mathrm{~cm}^{-1}$. Whereas the benzyl radical exhibited the origin band at $22002 \mathrm{~cm}^{-1}$, substitution of the methyl group(s) on the benzene ring always presented red-shifted origin bands, as listed in Table 1. For methylbenzyl radicals, the $o-, m$-, and $p$-xylyl radicals ${ }^{14}$ showed the origin bands at 21345,21485 , and $21700 \mathrm{~cm}^{-1}$, and were shifted by 657,517 , and $302 \mathrm{~cm}^{-1}$ from the benzyl radical, respectively, in the $\mathrm{D}_{1} \rightarrow \mathrm{D}_{0}$ electronic transition. For dimethylbenzyl radicals, the origin bands were observed in the region as predicted by the substitution effect which had been used to identify the isomers generated from the precursor in a corona discharge. The large shift in dimethylbenzyl radicals with substitution at the ortho position is related to the $o$-xylyl radical that showed the largest shift among the isomers.

Since the vibronic emission spectrum observed in a CESE system using a pinhole-type nozzle is similar to the dispersed fluorescence spectrum obtained by exciting the origin band of the electronic transition, the spacing of the vibronic bands from the origin band should represent the vibrational mode frequencies at the ground electronic state. Moreover, the origin band consistently showed a very strong intensity because of the highly efficient vibrational relaxation process in the $\mathrm{D}_{1}$ state and the similarity in molecular geometry between the two electronic states.

The spectrum showed several strong bands at 19088, $19538,20352,20800$, and $20836 \mathrm{~cm}^{-1}$ for the candidates of the origin band of each isomer and atomic lines belonging to hydrogen generated from dissociation of the $\mathrm{C}-\mathrm{H}$ bond and helium used for a carrier gas. 
Table 1. Origin Bands in the $D_{1} \rightarrow D_{0}$ Transition of MethylSubstituted Benzyl Radicals ${ }^{a}$

\begin{tabular}{|c|c|c|}
\hline Molecules & Origin band $\left(\mathrm{cm}^{-1}\right)$ & Shift $\left(\mathrm{cm}^{-1}\right)^{i}$ \\
\hline benzyl $^{b}$ & 22002 & 0 \\
\hline$o$-methylbenzyl ${ }^{c}$ & 21345 & 657 \\
\hline$m$-methylbenzyl ${ }^{c}$ & 21485 & 517 \\
\hline$p$-methylbenzyl ${ }^{c}$ & 21700 & 302 \\
\hline 2,3-dimethylbenzyl ${ }^{d}$ & 20657 & 1345 \\
\hline 2,4-dimethylbenzyl ${ }^{d}$ & 20600 & 1402 \\
\hline 2,5-dimethylbenzyl ${ }^{d}$ & 20558 & 1444 \\
\hline 2,6-dimethylbenzyl ${ }^{d}$ & 20616 & 1386 \\
\hline 3,4-dimethylbenzyl ${ }^{d}$ & 21306 & 696 \\
\hline 3,5-dimethylbenzyl ${ }^{e}$ & 20842 & 1160 \\
\hline 2,4,5-trimethylbenzyl $f^{f}$ & 20832 & 1170 \\
\hline 2,3,4-trimethylbenzyl ${ }^{g}$ & 20844 & 1158 \\
\hline 2,3,6-trimethylbenzyl ${ }^{g}$ & 19796 & 2206 \\
\hline 2,4,6-trimethylbenzyl ${ }^{h}$ & 20836 & 1166 \\
\hline 3,4,5-trimethylbenzyl $l^{h}$ & 20800 & 1202 \\
\hline 2,3,5-trimethylbenzyl ${ }^{h}$ & 19536 & 2466 \\
\hline
\end{tabular}

${ }^{a}$ Measured in vacuum $\left(\mathrm{cm}^{-1}\right) .{ }^{b}$ Reference $13 .{ }^{c}$ Reference $14 .{ }^{d}$ References 28-30. ${ }^{e}$ Reference $15 .{ }^{~}$ Reference $16 .{ }^{g}$ Reference $27 .{ }^{h}$ This work. ${ }^{i}$ With respect to the origin band of benzyl radical $\left(22002 \mathrm{~cm}^{-1}\right)$

To assign the vibronic bands belonging to each isomer, a series of vibrational structure of modes $\mathbf{6 a}, \mathbf{6 b}$, and $\mathbf{1}$ was checked in the spectrum because these are well-known vibrational modes in benzyl-type radicals. ${ }^{26}$ The in-plane C$\mathrm{C}-\mathrm{C}$ ring deformation vibrational modes $\mathbf{6 a}$ and $\mathbf{6 b}$ are degenerate at $606 \mathrm{~cm}^{-1}$ in benzene. With substitution on the benzene ring, these modes are split, providing lower and higher vibrational frequencies for modes $\mathbf{6 a}$ and $\mathbf{6 b}$, respectively, for $C_{2 \mathrm{v}}$ symmetry species, while the trend is reversed for $\mathrm{C}_{\mathrm{s}}$ symmetry. The precursor 1,2,3,5-tetramethylbenzene showed bands at 453 and $514 \mathrm{~cm}^{-1}$ for modes $\mathbf{6 a}$ and $\mathbf{6 b}$, respectively. The splitting between these two vibrational modes increases with increasing size of the substituents on the benzene ring. Other tetramethylbenzenes exhibit a comparable splitting between the two modes. Vibrational mode 1 of ring breathing is also the most eminent evidence for identification of aromatic compounds and is less sensitive to the substitution.

While the very strong bands at 19536, 20800, and 20836 $\mathrm{cm}^{-1}$ as an origin band show a series of vibrational structures of well-known vibrational modes, the bands at 19088 and $20352 \mathrm{~cm}^{-1}$ did not have any series of vibrational structure, suggesting that they were not the origin bands of the isomers. Thus, the bands at 19536, 20800, and $20836 \mathrm{~cm}^{-1}$ were assigned to be the origin bands of 2,3,5-, 3,4,5-, and 2,4,6-trimethylbenzyl radicals, respectively, produced from the precursor, according to the previous assignment ${ }^{2}$ in the solid state. In comparison with those measured in the solid state, the origin bands of jet-cooled 3,4,5- and 2,3,5-isomers showed a slightly increasing frequency than those in the solid state. However, the 2,4,6-isomer showed the origin band at a frequency lower by $133 \mathrm{~cm}^{-1}$ than that measured in the solid state. In the previous observation of benzyl-type radicals, ${ }^{27}$ two isomers, 2,3,4- and 2,3,6-trimethylbenzyl radicals, were formed from the 1,2,3,4-tetramethylbenzene, and the origin bands of each isomer showed very good agreement with that reported ${ }^{2}$ in the solid phase, shifted by about $500 \mathrm{~cm}^{-1}$. We strongly believe that this difference is tolerable in terms of the limited resolution and congested pattern with other small fragments produced in the photolysis. Of the three isomers, 3,4,5-isomer showed the strongest emission intensity in the spectrum, which can be explained by the larger transition dipole moment in the electronic transition, as verified in the TD-DFT calculation.

The observation of vibronic bands belonging to modes $\mathbf{6 a}$ and $\mathbf{1}$ was in a good agreement with those obtained in the

Table 2. Vibrational Mode Frequencies of Trimethylbenzyl Radicals ${ }^{a}$

\begin{tabular}{|c|c|c|c|c|c|}
\hline Mode $^{b}$ & $\begin{array}{l}\text { This work } \\
\left(\mathrm{D}_{0}\right)\end{array}$ & $\begin{array}{c}a b \text { initio }^{c} \\
\text { DFT/6-311G** }^{*}\left(\mathrm{D}_{0}\right)\end{array}$ & $\begin{array}{l}\text { Previous work }{ }^{d} \\
\qquad\left(\mathrm{D}_{0}\right)\end{array}$ & $\begin{array}{c}\text { Precursor } \\
1,2,3,5 \text {-tetramethylbenzene }{ }^{e}\left(\mathrm{~S}_{0}\right)\end{array}$ & Symmetry \\
\hline 2,3,5-trimethylbenzyl & & & & & $\mathrm{C}_{\mathrm{s}}$ \\
\hline $6 \mathbf{a}$ & 426 & 451 & & 453 & $a^{\prime}$ \\
\hline $6 \mathbf{b}$ & & 501 & & 514 & $a^{\prime}$ \\
\hline 1 & 574 & 597 & & 573 & $a^{\prime}$ \\
\hline 3,4,5-trimethylbenzyl & & & & & $C_{2 \mathrm{v}}$ \\
\hline origin & 20800 & & 20399 & & \\
\hline $6 \mathbf{a}$ & 448 & 457 & & 453 & $a_{1}$ \\
\hline $6 \mathbf{b}$ & & 501 & & 514 & $\mathrm{~b}_{2}$ \\
\hline 1 & 564 & 591 & & 573 & $a_{1}$ \\
\hline 2,4,6-trimethylbenzyl & & & & & $C_{2 \mathrm{v}}$ \\
\hline $6 \mathbf{a}$ & 450 & 454 & & 453 & $a_{1}$ \\
\hline $6 \mathbf{b}$ & & 503 & & 514 & $b_{2}$ \\
\hline 1 & 564 & 571 & & 573 & $a_{1}$ \\
\hline
\end{tabular}

${ }^{a}$ Measured in vacuum $\left(\mathrm{cm}^{-1}\right) .{ }^{b}$ Reference $31 .{ }^{c}$ Not scaled. ${ }^{d}$ Reference $2 .{ }^{e}$ Reference 26. 
Table 3. List of the Observed Vibronic Bands and Their Assignments

\begin{tabular}{|c|c|c|c|}
\hline Position $^{a}$ & Intensity & $\begin{array}{c}\text { Spacing from the } \\
\text { origin }^{b}\end{array}$ & Assignments $^{c}$ \\
\hline 21217 & $\mathrm{w}$ & & He atomic \\
\hline 20836 & s & $0(1)$ & origin of $2,4,6(1)$ \\
\hline 20800 & vs & $0(2)$ & origin of $3,4,5(2)$ \\
\hline 20764 & $\mathrm{w}$ & $36(2)$ & $\alpha(2)$ \\
\hline 20574 & $\mathrm{~m}$ & & $\mathrm{H}$ atomic \\
\hline 20410 & $\mathrm{~m}$ & $426(1)$ & $6 a_{1}^{0}(1)$ \\
\hline 20376 & vw & $460(1)$ & $\alpha(1)$ \\
\hline 20352 & vs & $448(2)$ & $6 a_{1}^{0}(2)$ \\
\hline 20316 & $\mathrm{w}$ & & $\alpha(2)$, He atomic \\
\hline 20262 & $\mathrm{~m}$ & $574(1)$ & $1_{1}^{0}(1)$ \\
\hline 20236 & $\mathrm{~m}$ & $564(2)$ & $1_{1}^{0}(2)$ \\
\hline 20106 & $\mathrm{~m}$ & $694(2)$ & $4_{1}^{0}(2)$ \\
\hline 20028 & $\mathrm{w}$ & $772(2)$ & $3_{1}^{0} 6 a_{1}^{0}(2)$ \\
\hline 19984 & $\mathrm{~m}$ & $852(1)$ & $6 a_{2}^{0}(1)$ \\
\hline 19940 & $\mathrm{~s}$ & & He atomic \\
\hline 19902 & $\mathrm{~m}$ & $898(2)$ & $6 a_{2}^{0}(2)$ \\
\hline 19842 & w & $994(1)$ & $1_{1}^{0} 6 a_{1}^{0}(1)$ \\
\hline 19812 & w & & He atomic \\
\hline 19786 & $\mathrm{~m}$ & 1014(2) & $1_{1}^{0} 6 a_{1}^{0}(2)$ \\
\hline 19718 & $\mathrm{~m}$ & $1118(1)$ & $1_{2}^{0}(1)$ \\
\hline 19680 & $\mathrm{vw}$ & $1120(2)$ & $1_{2}^{0}(2)$ \\
\hline 19582 & $\mathrm{vw}$ & $1218(2)$ & $3_{1}^{0} 6 a_{2}^{0}(2)$ \\
\hline 19536 & $\mathrm{~s}$ & $0(3)$ & origin of $2,3,5(3)$ \\
\hline 19502 & $\mathrm{vw}$ & $36(3)$ & $\alpha(3)$ \\
\hline 19378 & $\mathrm{w}$ & $1458(1)$ & $1_{1}^{0} 6 a_{2}^{0}(1)$ \\
\hline 19342 & $\mathrm{vw}$ & 1494(1) & $\alpha(1)$ \\
\hline 19276 & $\mathrm{~m}$ & $262(3)$ & $9 a_{1}^{0}(3)$ \\
\hline 19242 & w & 296(3) & $9 b_{1}^{0}(3)$ \\
\hline 19212 & $\mathrm{w}$ & $326(3)$ & $3_{1}^{0}(3)$ \\
\hline 19088 & $\mathrm{~s}$ & $450(3)$ & $6 a_{1}^{0}(3)$ \\
\hline 19052 & $\mathrm{w}$ & $486(3)$ & $\alpha(3)$ \\
\hline 18974 & $\mathrm{w}$ & $564(3)$ & $1_{1}^{0}(3)$ \\
\hline 18828 & $\mathrm{w}$ & $710(3)$ & $4_{1}^{0}(3)$ \\
\hline 18762 & w & $776(3)$ & $3_{1}^{0} 6 a_{1}^{0}(3)$ \\
\hline 18638 & w & $900(3)$ & $6 a_{2}^{0}(3)$ \\
\hline 18416 & w & $1122(3)$ & $1_{2}^{0}(3)$ \\
\hline
\end{tabular}

${ }^{a}$ Measured in vacuum $\left(\mathrm{cm}^{-1}\right) .{ }^{b}$ The numbers 1,2 , and 3 in parentheses indicate the spacing from the origin band in the $\mathrm{D}_{1} \rightarrow \mathrm{D}_{0}$ transition of the 2,4,6-, 3,4,5-, and 2,3,5-trimethylbenzyl radicals, respectively. ${ }^{c}$ The numbers 1,2 , and 3 in parentheses indicate the bands belonging to the $\mathrm{D}_{1}$ $\rightarrow \mathrm{D}_{0}$ transition of the 2,4,6-, 3,4,5-, and 2,3,5-trimethylbenzyl radicals, respectively. The Greek letter indicates the torsional bands belonging to the strong bands.

calculation for three isomers. The slight reduction of $\mathbf{6 a}$ mode in the 2,3,5-isomer was attributed to the change of symmetry from $C_{2 \mathrm{v}}$ to $\mathrm{C}_{\mathrm{s}}$. Table 2 compares the vibrational mode frequencies of trimethylbenzyl radicals for modes $\mathbf{6 a}$, $\mathbf{6 b}$, and $\mathbf{1}$ with the calculated values and those of the precursor.

Following the determination of the origin bands of each isomer, other vibronic bands were straightforwardly assigned for each species by comparing with the calculated values and those of the precursor. From the assignments, relatively long progressions involving the vibrational modes $\mathbf{1}$ and $\mathbf{6 a}$ were observed from the emission spectrum for all three isomers. The observations were in excellent agreement with those of calculation without any scaling. Short progressions involving the vibrational mode $4\left(694 \mathrm{~cm}^{-1}\right)$ of 3,4,5-isomer and the modes $9 \mathbf{a}\left(262 \mathrm{~cm}^{-1}\right), 9 \mathbf{b}\left(296 \mathrm{~cm}^{-1}\right), \mathbf{3}\left(326 \mathrm{~cm}^{-1}\right)$, and $4\left(710 \mathrm{~cm}^{-1}\right)$ of 2,3,5-isomer were determined. The modes listed above were also active in combination bands. The vibrational mode frequencies obtained in this work are listed in Table 3, together with the identification of the isomers. In addition to the vibronic bands assigned, we observed a few weak bands in the interval of about $36 \mathrm{~cm}^{-1}$ in the vicinity of a very strong vibronic band, which suggests that these belong to torsional transitions of the methyl group during jet expansion. ${ }^{11}$

In summary, the vibronic emission spectrum of the benzyl-type radicals in the $\mathrm{D}_{1} \rightarrow \mathrm{D}_{0}$ transition were recorded in the visible region with a CESE system from the corona discharge of the precursor 1,2,3,5-tetramethylbenzene seeded in a large amount of inert carrier gas helium using a pinholetype glass nozzle. A comparison with the data obtained from $a b$ initio calculations using the Gaussian program revealed evidence of three isomeric trimethylbenzyl radicals generated in the corona discharge of the precursor. The origin bands and other vibronic bands belonging to these three isomers were clearly identified by comparison with those from $a b$ initio calculations, as well as those from the known data of the precursor.

Acknowledgments. This work was supported by the National Research Foundation of Korea (NRF) grant funded by the Korea Government (MEST) (No. 2011-0011009 ).

\section{References}

1. Tan, X. Q.; Wright, T. G.; Miller, T. A. In Jet Spectroscopy and Molecular Dynamics; Hollas, J. M., Philip, D., Eds.; Blackie: London, 1994.

2. Branciard-Larcher, C; Migirdicyan, E. Chem. Phys. 1973, 2, 95.

3. Schuler, H.; Reinbeck, L.; Kaberle, A. R. Z. Naturforsh 1952, 7A, 421.

4. Walker, S.; Barrow, R. F. Trans. Faraday Soc. 1954, 50, 541.

5. Bindley, T. F.; Watts, A. T.; Watts, S. Trans. Faraday Soc. 1962, 58,849 .

6. Bindley, T. F.; Watts, A. T.; Watts, S. Trans. Faraday Soc. 1964, $60,1$.

7. Carlton, T. R.; Thrush, B. A. Chem. Phys. Lett. 1986, 125, 547.

8. Hiratsuka, H.; Mori, K.; Shizuke, H.; Fukushima, M.; Obi, K. Chem. Phys. Lett. 1989, 157, 35.

9. Cossart-Magos, C.; Leach, S. J. Chem. Phys. 1976, 64, 4006.

10. Fukushima, M.; Obi, K. J. Chem. Phys. 1990, 93, 8488.

11. Suh, M. H.; Lee, S. K.; Miller, T. A. J. Mol. Spectrosc. 1999, 194, 211

12. Lin, T.-Y. D.; Miller, T. A. J. Phys. Chem. 1990, 94, 3554.

13. Selco, J. I.; Carrick, P. G. J. Mol. Spectrosc. 1989, 137, 13.

14. Selco, J. I.; Carrick, P. G. J. Mol. Spectrosc. 1995, 173, 277.

15. Lee, G. W.; Lee, S. K. J. Phys. Chem. A 2007, 111, 6003.

16. Lee, G. W.; Lee, S. K. J. Chem. Phys. 2007, 126, 214308.

17. Petruska, J. J. Chem. Phys. 1961, 34, 1111.

18. Lee, S. K. Chem. Phys. Lett. 2002, 358, 110.

19. Han, M. S.; Choi, I. S.; Lee, S. K. Bull. Korean Chem. Soc. 1996, 
17, 991.

20. Weise, M. L.; Smith, M. W.; Glennon, B. M. Atomic transition Probabilities; NSRD-NBS4; NBS: Gaithsburg, MD, 1966.

21. Cossart-Magos, C.; Cossart, D. Mol. Phys. 1988, 65, 627.

22. Banwell, C. N.; McCash, E. M. Fundamentals of Molecular Spectroscopy, $4^{\text {th }}$ ed.; McGraw-Hill: New York, NY, 1994.

23. Fujiwara, M.; Tanimoto, Y. J. Phys. Chem. 1994, 98, 5695.

24. Lejeune, V.; Despres, A.; Fourmann, B.; Benoist d'Azy, O.; Migirdicyan, E. J. Phys. Chem. 1987, 91, 6620.

25. Lee, S. K.; Baek, D. Y. Chem. Phys. Lett. 1999, 304, 39.
26. Varsanyi, G. Assignments for Vibrational Spectra of Seven Hundred Benzene Derivatives; John Wiley \& Sons: New York, NY 1974.

27. Yoon, Y. W.; Lee, G. W.; Lee, S. K. Bull. Korean Chem. Soc. submitted.

28. Lee, G. W.; Lee, S. K. J. Phys. Chem. A 2006, 110, 2130.

29. Lee, G. W.; Lee, S. K. J. Phys. Chem. A 2006, 110, 1812.

30. Ahn, H. G.; Lee, G. W.; Kim, T. K.; Lee, S. K. Bull. Korean Chem. Soc. 2008, 29, 2341.

31. Wilson, E. B. Phys. Rev. 1934, 45, 706. 